\title{
DNA imaged on a HOPG electrode surface by AFM with controlled potential
}

\author{
Ana Maria Oliveira Brett*, Ana-Maria Chiorcea Paquim \\ Departamento de Química, Faculdade de Ciências e Tecnologia, Universidade de Coimbra, 3004-535 Coimbra, Portugal
}

Received 12 December 2003; received in revised form 27 April 2004; accepted 5 May 2004

Available online 26 August 2004

\begin{abstract}
Single-molecule AFM imaging of single-stranded and double-stranded DNA molecules self-assembled from solution onto a HOPG electrode surface is reported. The interaction of DNA with the hydrophobic surface induced DNA aggregation, overlapping, intra- and intermolecular interactions. Controlling the electrode potential and using the phase images as a control method, to confirm the correct topographical characterization, offers the possibility to enlarge the capability of AFM imaging of DNA immobilized onto conducting substrates, such as HOPG. The application of a potential of $+300 \mathrm{mV}$ (versus AgQRE) to the HOPG enhanced the robustness and stability of the adsorbed DNA molecules, increasing the electrostatic interaction between the positively charged electrode surface and the negatively charged DNA sugar-phosphate backbone.
\end{abstract}

(C) 2004 Elsevier B.V. All rights reserved.

Keywords: DNA; Adsorption; HOPG; Atomic force microscope; AFM

\section{Introduction}

DNA is an important biomacromolecule with remarkable chemical and biophysical properties [1-3]. The adsorption of single-stranded and double-stranded DNA at the solid electrodes surface plays a vital role in a variety of biotechnological, medical and nanoscience applications and enables the chemical and structural modification of the sensor surface [4], being very important for understanding many physiological processes. Different structures and conformations that DNA molecules can adopt at the electrode surface lead to different interactions with other molecules, such as modifications of the accessibility of different drugs to the DNA grooves and modifications in DNA hybridization efficiency.

Atomic force microscopy (AFM) has proved to be a powerful tool for obtaining high-resolution images of DNA in air and in solution. Images of DNA conformations,

\footnotetext{
* Corresponding author. Tel./fax: +351 239835295.

E-mail address: brett@ci.uc.pt (A.M. Oliveira Brett).
}

unusual structures and DNA-protein complexes have been obtained almost exclusively on mica or silicon [5-8], but rarely on conducting materials. Effectively, the DNA molecules do not bind strongly enough to conducting substrates and the AFM tip tends to sweep away the adsorbed macromolecules. AFM imaging onto conducting substrates has been limited to gold substrates [9]. However, the oxidation of the gold electrodes occurs at potentials of approximately $+0.8 \mathrm{~V},[10,11]$ and the gold surface becomes covered with gold oxides. Electrochemical oxidation of nucleic acids on carbon electrodes showed that, with the exception of guanine base, which has an oxidation peak at approximately $+0.8 \mathrm{~V}$, depending on the experimental conditions and electrodes used, all the other nucleic acid bases and nucleosides are oxidized at higher electrode potentials $[12,13]$. Consequently, the gold surface is not a good choice for electrochemical studies of DNA due to its limited potential range. A major challenge in the area of direct visualization of DNA molecules is to extend the capability of AFM imaging to other conducting substrates required in electrochemical applications. 
Carbon electrodes such as glassy carbon, carbon fibres, graphite or carbon black have a wide useful potential range, particularly in the positive direction, which enables the detection of damage caused to DNA by following the oxidation peaks of the purine bases [12-14].

Highly oriented pyrolytic graphite (HOPG) has been used to study single DNA molecules by STM. However, the geometry of the grain boundary, the step edge texture and surface defects of the freshly cleaved HOPG surface produce STM images that mimic both single-stranded and double-stranded DNA molecules, interfering with the ability to distinguish between the true biological features and the features related with the clean HOPG steps [15-17]. The problems encountered in STM imaging of the molecules adsorbed onto HOPG steps also limited the use of HOPG in AFM studies of single-DNA molecules. AFM has been has been limited to studying the formation of DNA networked films assembled from high and very high DNA solution concentrations [18-20], which enabled the formation of thick and stable DNA lattices, completely covering the HOPG boundary defects. Nevertheless, the nature of the single DNA-surface interaction and the morphology adopted by isolated DNA molecules when small solution concentrations are used is still not yet well understood.

Magnetic AC mode AFM (MAC Mode AFM) permits the visualization of the molecules weakly bound to the substrate material and it can be very helpful in the investigation of single-molecules loosely attached to the conducting surface of electrochemical transducers. AFM is not as sensitive as STM to unusual electronic structures on the surface, the AFM images being less affected by artefacts than STM [5].

In this context, this paper explores the possibility of using MAC Mode AFM to image single-stranded DNA (ssDNA) and double-stranded DNA (dsDNA) from calfthymus immobilized by free adsorption and by applying a potential of $+300 \mathrm{mV}$ vs. AgQRE to the HOPG electrode surface immersed in solutions of low DNA concentration. Calf-thymus DNA is a large molecule that is easily commercially available and has already been used for biosensor construction $[13,14]$.

\section{Materials and methods}

\subsection{Materials}

Calf-thymus double-stranded DNA (sodium salt, type I) and single-stranded DNA were purchased from SigmaAldrich Química, Spain and were used without further purification. The electrolyte used was $\mathrm{pH} \quad 7.0 \quad 0.1 \mathrm{M}$ phosphate buffer solution and was prepared using analytical grade reagents and purified water from a Millipore Milli-Q system (conductivity $<0.1 \mu \mathrm{S} \mathrm{cm}{ }^{-1}$ ). Solutions of different concentrations were obtained by direct dilution of the appropriate volume in phosphate buffer.
Highly oriented pyrolytic graphite (HOPG), grade $\mathrm{ZYH}$, of rectangular shape with $15 \times 15 \times 2 \mathrm{~mm}$ dimensions, from Advanced Ceramics, UK, was used throughout this study as a substrate. The HOPG was freshly cleaved with adhesive tape prior to each experiment and was imaged by Contact Mode AFM in order to establish its cleanliness.

Voltammetric experiments were carried out in a onecompartment Teflon cell of approximately $12.5 \mathrm{~mm}$ internal diameter holding the HOPG sample - the working electrode - on the base. A Pt wire counter electrode and a silver wire as quasi-reference (AgQRE) electrode were placed in the cell, dipping approximately $5 \mathrm{~mm}$ into the solution. The electrochemical control was done with a PalmSens potentiostat, running with PalmScan version 1.11 software, from Palm Instruments, The Netherlands.

\subsection{DNA sample preparation}

For DNA samples prepared by free adsorption, $100 \mu 1$ of DNA solutions were adsorbed onto freshly cleaved HOPG surface and incubated for $3 \mathrm{~min}$. The excess of DNA was gently cleaned with a jet of Milli Q water and the HOPG with adsorbed DNA was then dried with nitrogen, which is a typical procedure used for imaging dry nucleic acid molecules in air [6].

For DNA samples prepared by electrochemically assisted adsorption $500 \mu \mathrm{l}$ of the desired DNA solution were placed in the electrochemical cell holding the HOPG working electrode on the base. A positive potential of $300 \mathrm{mV}$ (vs. AgQRE) was applied to the electrode during $3 \mathrm{~min}$. The HOPG with adsorbed DNA was rinsed with a jet of Milli-Q water and dried with nitrogen.

\subsection{Atomic force microscopy}

AFM was performed with a Pico SPM controlled by a MAC Mode (Magnetic AC Mode) module and interfaced with a PicoScan controller from Molecular Imaging, USA. All the AFM experiments were performed with a CS AFM S scanner with the scan range $6 \mu \mathrm{m}$ in $x-y$ and $2 \mu \mathrm{m}$ in $z$, Molecular Imaging Corporation. Silicon type II MAClevers $225 \mu \mathrm{m}$ length, $2.8 \mathrm{~N} / \mathrm{m}$ spring constant and $60-90 \mathrm{kHz}$ resonant frequencies (Molecular Imaging) were used in MAC Mode AFM. Silicon nitride NanoProbes TM Vshaped cantilevers, $100 \mu \mathrm{m}$ length, $0.58 \mathrm{~N} / \mathrm{m}$ spring constant were used for contact AFM in air.

All images (256 samples/line $\times 256$ lines) were taken at room temperature, scan rates $1.0-1.3$ lines $\mathrm{s}^{-1}$. The images were processed by first order flattening in order to remove the background slope and the contrast and brightness were adjusted. All images were visualized in three-dimensions using the Scanning Probe Image Processor, SPIP version 2.3011, Image Metrology ApS. Section analysis over DNA molecules and films was performed with PicoScan software version 6.0, Molecular Imaging. 


\section{Results and discussion}

\subsection{Free adsorption of ssDNA and $d s D N A$ on $H O P G$}

DNA is a highly charged, hydrophilic molecule, whereas HOPG has a hydrophobic surface. These characteristics reduce the spontaneous interaction of DNA with the HOPG surface. Despite the fact that MAC mode AFM is a gentle technique, with a view to minimizing as much as possible any damage to the biological films by the AFM tip, it represents one of the most significant problems when imaging single DNA molecules. In order to improve the stability of the molecules on the surface, the DNA samples have been dried and imaged in air before being observed by AFM. This adsorption procedure, used in the AFM studies, is similar to the real procedure of preparation and storage of DNA-modified electrodes.

The AFM results indicate that both ssDNA and dsDNA adsorb freely at the HOPG surface and the adsorption is fast. The MAC Mode AFM topographical images of ssDNA adsorbed onto HOPG, from a $1 \mu \mathrm{g} / \mathrm{ml} \mathrm{ssDNA}$ solution in $\mathrm{pH}$

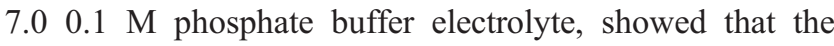
ssDNA molecules condensed at the surface in very large, coiled and twisted featureless aggregates, Fig. 1A. ssDNA has the bases exposed, which facilitates interaction with the substrate. The long ssDNA molecules exist in solution in flexible polymeric-like configurations with some hybridization of randomly complementary zones. The conformation of the molecules is inevitably modified by the transition from three dimensions in solution to two dimensions on the surface. The ssDNA molecules are stabilized on the HOPG surface by hydrophobic interactions between the hydrophobic aromatic rings of the bases and the hydrophobic carbon surface.

Scanning probe microscopy visualization of DNA as such small concentrations of molecules of $1 \mu \mathrm{g} / \mathrm{ml}$ requires a careful analysis of the geometry related with the HOPG domain walls, since the molecules are isolated and not actually form uniform films, not covering completely the HOPG surface. As demonstrated by STM studies, difficulty arise in distinguish between an HOPG step and an adsorbed molecule $[15,16]$.

In MAC Mode AFM, the parameters of AFM cantilever oscillations (amplitude, frequency, phase) change when the cantilever contacts the sample surface. A significant advantage of MAC Mode AFM, compared with contact mode AFM, is the possibility of using the changes in phase angle of the AFM cantilever probe to produce a second image, the phase image. As well as the amplitude image (feedback error), which is the equivalent of the deflection image in contact mode AFM, the phase image is recorded simultaneously with the topographical image of the surface, and represents the difference between the oscillating magnetic field driving the cantilever and its true response at surface contact. The reference phase is considered the free oscillation phase of the AFM cantilever, far away from the surface. During scanning of the sample, the changes in phase contrast depend not only on topography changes, but also on the adhesion, elasticity and viscoelastic properties of the surface. The modifications in the phase angle are correlated with different damping produced by different areas of the sample surface.

Frequently, the phase images show an improved contrast compared with the corresponding DNA topographical image [21], and can give a second opinion about the doubtful features in the HOPG sample and about the presence of DNA molecules.

In Fig. 1 are presented the amplitude image (feedback error), Fig. 1B, and the phase image, Fig. 1C, recorded simultaneously with the topographic image from Fig. 1A. The big ssDNA aggregate observed is marked by (1) in the images and an HOPG edge step between two graphite terraces is marked by (2). As can be observed in topographical and amplitude images, it is difficult to distinguish between the soft biological molecules and the HOPG border. However, DNA is much more viscous than the bare HOPG surface leading to a more pronounced difference in phase angle as the AFM tip passes over, Fig. 1C. Using the phase image, it is very easy to identify the real DNA molecules with an improved contrast and to distinguish them from the unusual HOPG structures, overcoming in this
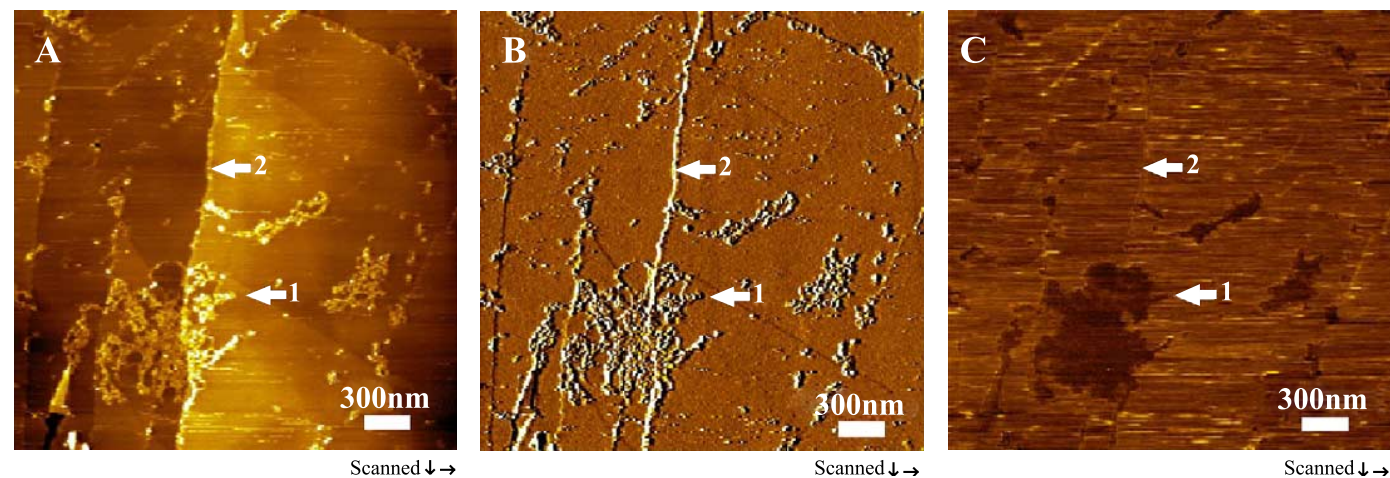

Fig. 1. (A) MAC Mode AFM topographical image in air of ssDNA prepared onto HOPG by 3 min free adsorption from $1 \mu \mathrm{g} / \mathrm{ml} \mathrm{ssDNA}$ solution in $\mathrm{pH} 7.00 .1$ M phosphate buffer electrolyte. (B) Amplitude image (feedback error) and (C) phase image recorded simultaneously with the topographic image A. Inside the images (1) marks an ssDNA aggregate and (2) marks an HOPG step. 
way the problems of image artefacts encountered in STM imaging of single DNA molecules.

MAC Mode AFM images in air of dsDNA self-assembled on $\mathrm{HOPG}$, from $1 \mu \mathrm{g} / \mathrm{ml}$ dsDNA solution in $\mathrm{pH} 7.00 .1 \mathrm{M}$ phosphate buffer electrolyte, also revealed coiled and twisted structures but the dsDNA molecules appeared more extended on the HOPG surface, Fig. 2A. Sporadically, sections of straight dsDNA could be observed as shown in Fig. $2 \mathrm{C}$. The phase images, Fig. 2B and D, represent a control method to certify that the topographical characterization is correct and it is really the DNA that is seen in the images.

The measured full width at half-maximum height (fwhm) of isolated dsDNA was approximately 10-25 nm, being overestimated due to the convolution effect of the tip radius.
The height measurements were not limited by the tip radius and give a better representation of the dsDNA diameter. Therefore, the straight portions of dsDNA offer the possibility of evaluating the true height of the dsDNA molecules. The average height measured by section analysis over parts of single dsDNA molecules in the images, Fig. $2 \mathrm{~F}$, was $0.7 \pm 0.2 \mathrm{~nm}$. It is expected that isolated dsDNA adopts the A-form due to strong dehydration of the molecules leading to a double-helix diameter of approximately $2.6 \mathrm{~nm}$. In AFM studies in air, the reported heights for dsDNA confined on a solid support vary from 0.5 to $1.9 \mathrm{~nm}$ [6], usually much smaller than the helix diameter, which probably due to elastic deformations of dsDNA caused by the AFM tip [6]. The large fwhm of dsDNA may
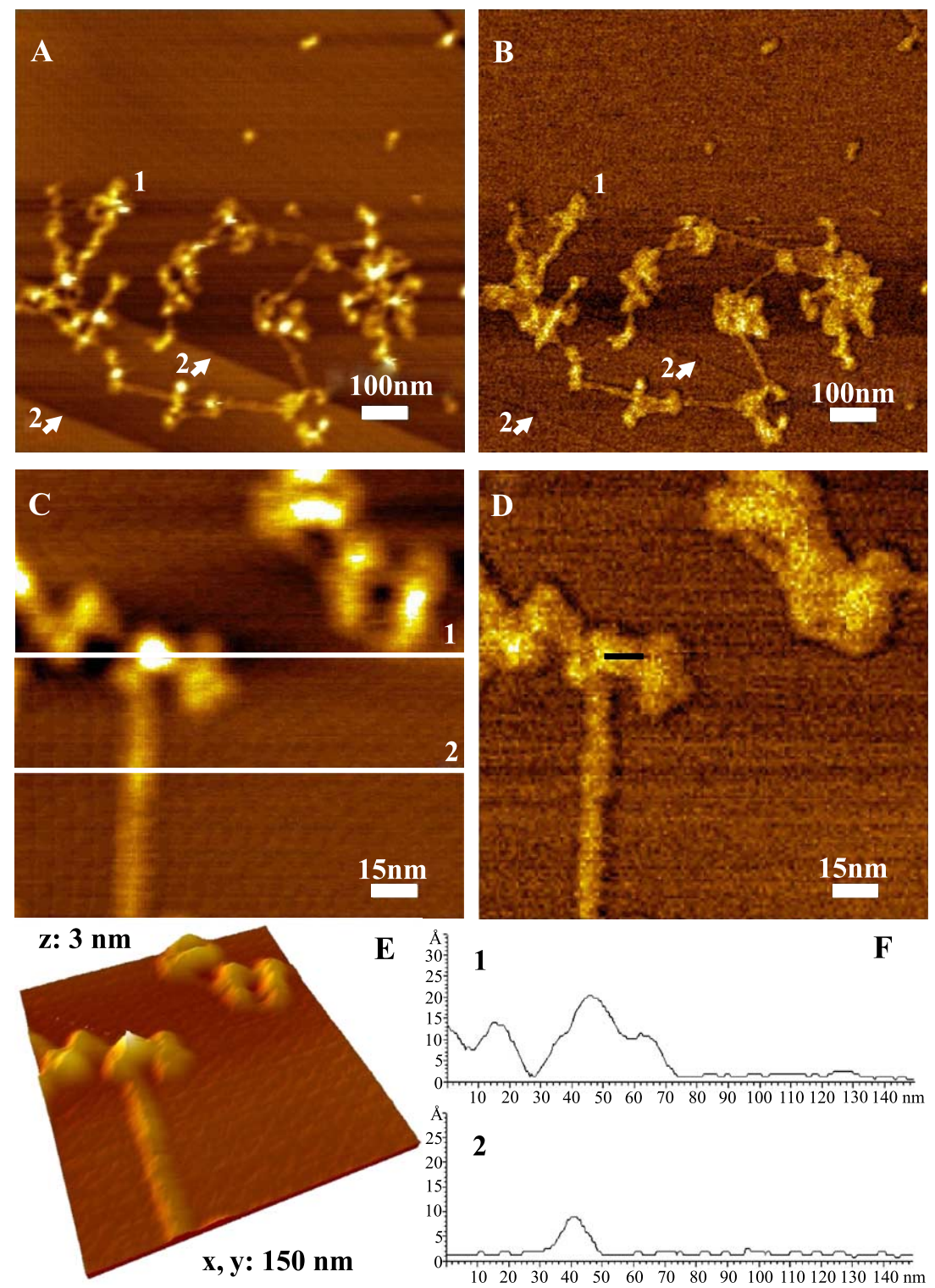

Fig. 2. (A, C) MAC Mode AFM topographical images in air of dsDNA prepared onto HOPG by 3 min free adsorption from $1 \mu \mathrm{g} / \mathrm{ml} \mathrm{dsDNA}$ solution in $\mathrm{pH} 7.0$ $0.1 \mathrm{M}$ phosphate buffer electrolyte. (B, D) Phase images recorded simultaneously with the topographic images A and C. Inside the images A and B (1) marks an dsDNA aggregate and (2) marks an HOPG step. (E) Three-dimensional representation of image C. (F) Cross-section profiles through white lines 1 and 2 in the image $\mathrm{C}$. 
be not only to an artefact caused by the size and shape of the tip, but also a broadening of dsDNA itself as a consequence of dsDNA deformation by the tip.

In the case of dsDNA, the aggregation phenomena were less critical than in the case of ssDNA, due to larger electrostatic repulsion between the negatively charged sugar-phosphate backbone of the molecule strands. However, it was noticed that the dsDNA displayed spherical aggregates along its length with sizes between 1 and $2.5 \mathrm{~nm}$, the $150 \times 150 \mathrm{~nm}$ scan size image. The presence of salt in the buffer electrolyte can induce spontaneous condensation of dsDNA due to lateral aggregation of the molecule [1]. Also, the hydrophobic HOPG surface may induce aggregation of long dsDNA, even in the absence of buffer solution. At the solid-liquid interface, the dsDNA environment is drastically different from that in bulk solution since DNA is forced to pass from the solution $3 \mathrm{D}$ conformation to the adsorbed $2 \mathrm{D}$ conformation. The drying procedure does not modify the adsorbed double- or single-stranded DNA on the HOPG surface but the aggregates of the dehydrated 2D conformation are less high.

Adsorption and aggregation at other hydrophobic polymeric surfaces [22,23] was observed. In the double-helical structure, a continuous dissociation-association of the bases of dsDNA occurs at the ends as well as at single-stranded overhangs ("sticky ends") [1-3]. The stabilization of dsDNA at the surface may occur through interaction between the hydrophobic HOPG surface and several hydrophobic bases at the dsDNA ends. The interaction of dsDNA with the HOPG surface can induce overlapping and superposition of the molecules, sticky-ended cohesion and conformation changes, leading to DNA-DNA interactions and to formation of alternative DNA structures [2,3,23].

\subsection{Adsorption of SsDNA and dsDNA on HOPG under an applied potential}

The immobilization of ssDNA and dsDNA by free adsorption onto HOPG is very quick and easy to perform. The main disadvantage of this method is that the nucleic acids cluster into very large aggregates, especially in the case of ssDNA.

Nucleic acid self-assembly involves a large number of weak interactions and the DNA molecules do not bind strongly enough to the conducting HOPG substrate. Consequently, the DNA molecules are rather unstable on the surface and may be desorbed. The friction caused by the AFM tip during scanning the surface is frequently superior to the adhesion to the surface and the AFM tip easily sweeps away and drags the DNA molecules adsorbed on the HOPG surface. It was observed during the experiments that the AFM tip could easily move fragments of DNA molecules condensed by free adsorption. Fig. 3 shows an example of how the AFM tip causes the dislocation and movement of ssDNA fragments along the HOPG surface; this image was recorded immediately after the images from Fig. 1. In all three images (topography Fig. 1A, amplitude Fig. 1B, phase Fig. 1C), traces of the trajectories of these fragments clearly appear.

During free adsorption, the single DNA molecules are preferentially attached near the step edges of the HOPG surface, Figs. 1A and 2A. A clean, atomically flat surface is produced by peeling the top layer of the HOPG basal cleavage plane. This process induces the formation of different structures on graphite, e.g., grain boundaries, dislocations, cleavage steps and point defects [17]. The non-bound carbon atoms existing on these defects may form bonds with hydrogen, hydroxyl and carboxyl groups [24]. These functional groups formed at the HOPG steps as well as breaks in the surface may contribute to the predisposition of nucleic acid molecules to accumulate at these sites.

In order to overcome all these problems, a stronger and controlled adsorption of DNA onto the HOPG surface is required. A better immobilization was obtained by applying a positive potential to the surface during adsorption. The positively charged HOPG substrate exerts electrostatic attraction on the nucleic acid molecules. The potential chosen was $+300 \mathrm{mV}$, versus AgQRE, during $3 \mathrm{~min}$, because this potential is not positive enough to irreversibly oxidize the DNA bases inside the molecules [12] and was
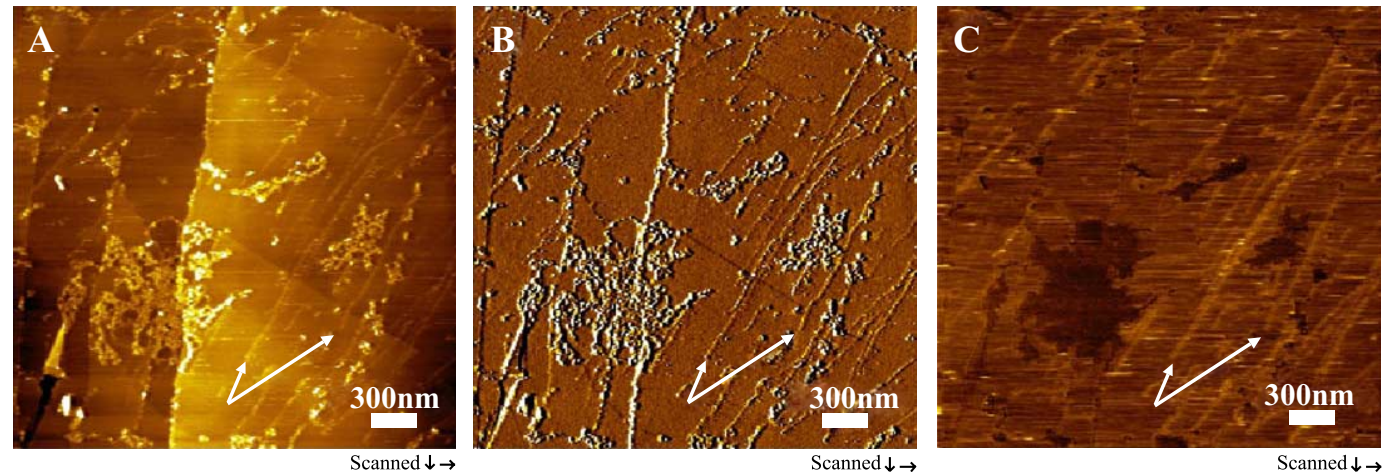

Fig. 3. (A) MAC Mode AFM topographical image in air of ssDNA prepared onto HOPG by 3 min free adsorption from $1 \mu \mathrm{g} / \mathrm{ml} \mathrm{ssDNA}$ solution in $\mathrm{pH} 7.00 .1$ $\mathrm{M}$ phosphate buffer electrolyte. (B) Amplitude image (feedback error) and (C) phase image recorded simultaneously with the topographic image C. Inside the images, the arrows mark the trajectory traces of ssDNA fragments moved by the AFM tip. 
considered to be sufficient to stabilize the negatively charged DNA molecules on the surface.

We have found that electrochemical adsorption is very efficient in the case of ssDNA. At concentrations of $1 \mu \mathrm{g} / \mathrm{ml}$ ssDNA solutions in $\mathrm{pH} \quad 7.0 \quad 0.1 \mathrm{M}$ phosphate buffer electrolyte, a two-dimensional network was formed by linear strands covering the entire HOPG surface, Fig. 4. Variable angles between the network strands could be observed and the height of the strands was $1.4 \pm 0.3 \mathrm{~nm}$. At the branch junctions and along the linear segments of the lattice, many globular clusters appeared with height between 2 and $4 \mathrm{~nm}$. This may be due to crossover molecules or to binding of linear strands while forming the lattice. It is also very probable that many parts of the ssDNA molecules contain complementary bases leading to local hybridization and the formation of portions of dsDNA. A network formation at the HOPG electrode surface was also observed for different concentrations of ssDNA.

The dsDNA molecules adsorbed by deposition at +300 $\mathrm{mV}$ versus $\mathrm{AgQRE}$, from a solution of $1 \mu \mathrm{g} / \mathrm{ml}$ dsDNA in pH 7.0 0.1 M phosphate buffer electrolyte, Fig. 5, also presented higher stability on the surface compared with dsDNA immobilized by free adsorption. Still the applied potential is less effective than in the case of ssDNA, being not able to prevent the aggregation of the molecules. In aqueous solution, the dsDNA has the negatively charged phosphate groups distributed helically outside the duplex. During the controlled potential adsorption process, dsDNA adsorbs electrostatically at a given number of points along its length. The dsDNA had a large number of intramolecular
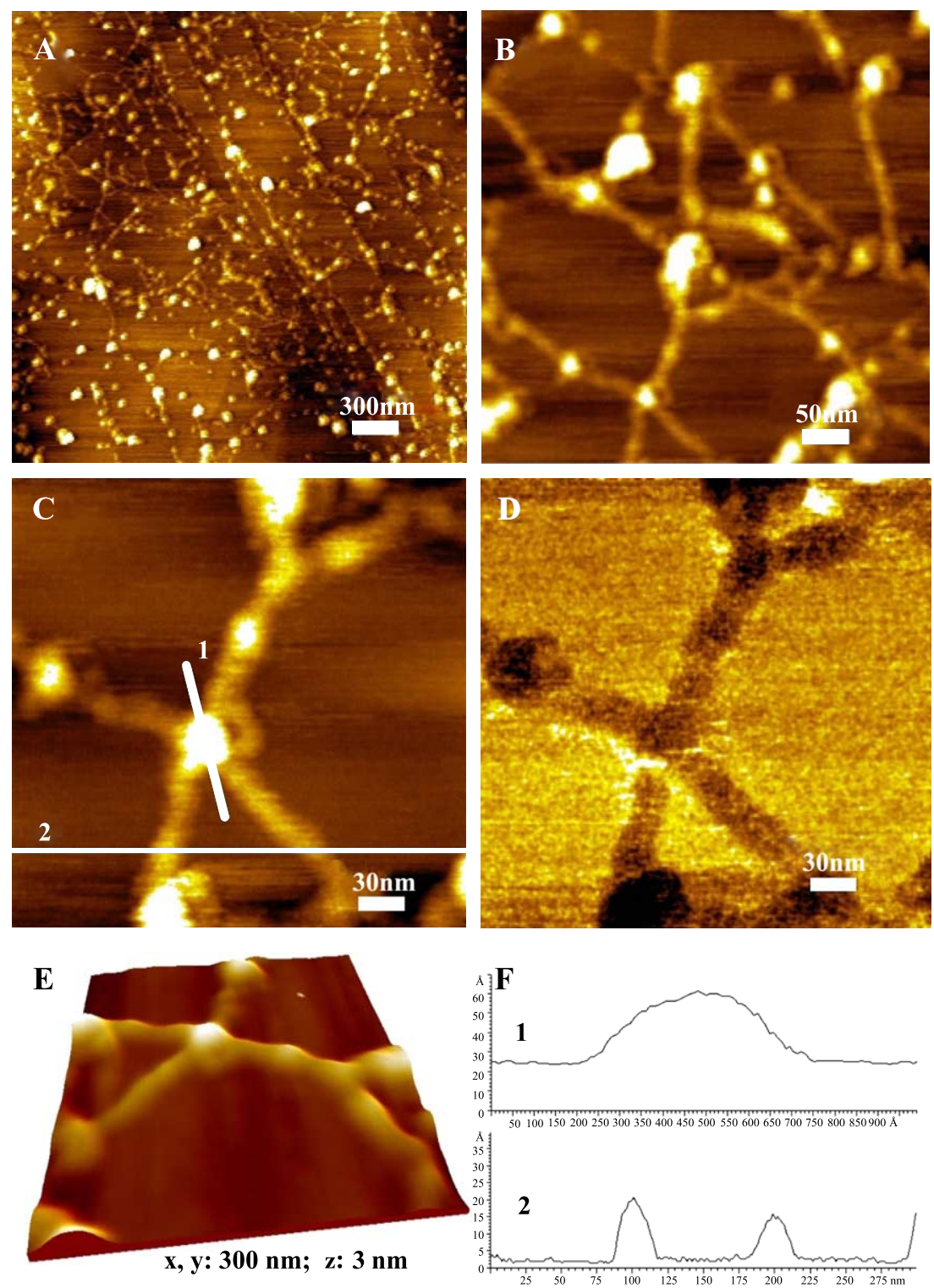

Fig. 4. (A, B, C) MAC Mode AFM topographical images in air of ssDNA prepared onto HOPG by applying a deposition potential of $+300 \mathrm{mV}$ (vs. AgQRE) during 3 min to the electrode immersed into $1 \mu \mathrm{g} / \mathrm{ml}$ ssDNA solution $\mathrm{pH}$ 7.0 0.1 M phosphate buffer electrolyte. (D) Phase image recorded simultaneously with the topographic image C. (E) Three-dimensional representation of image C. (F) Cross-section profiles through white lines 1 and 2 in the image C. 

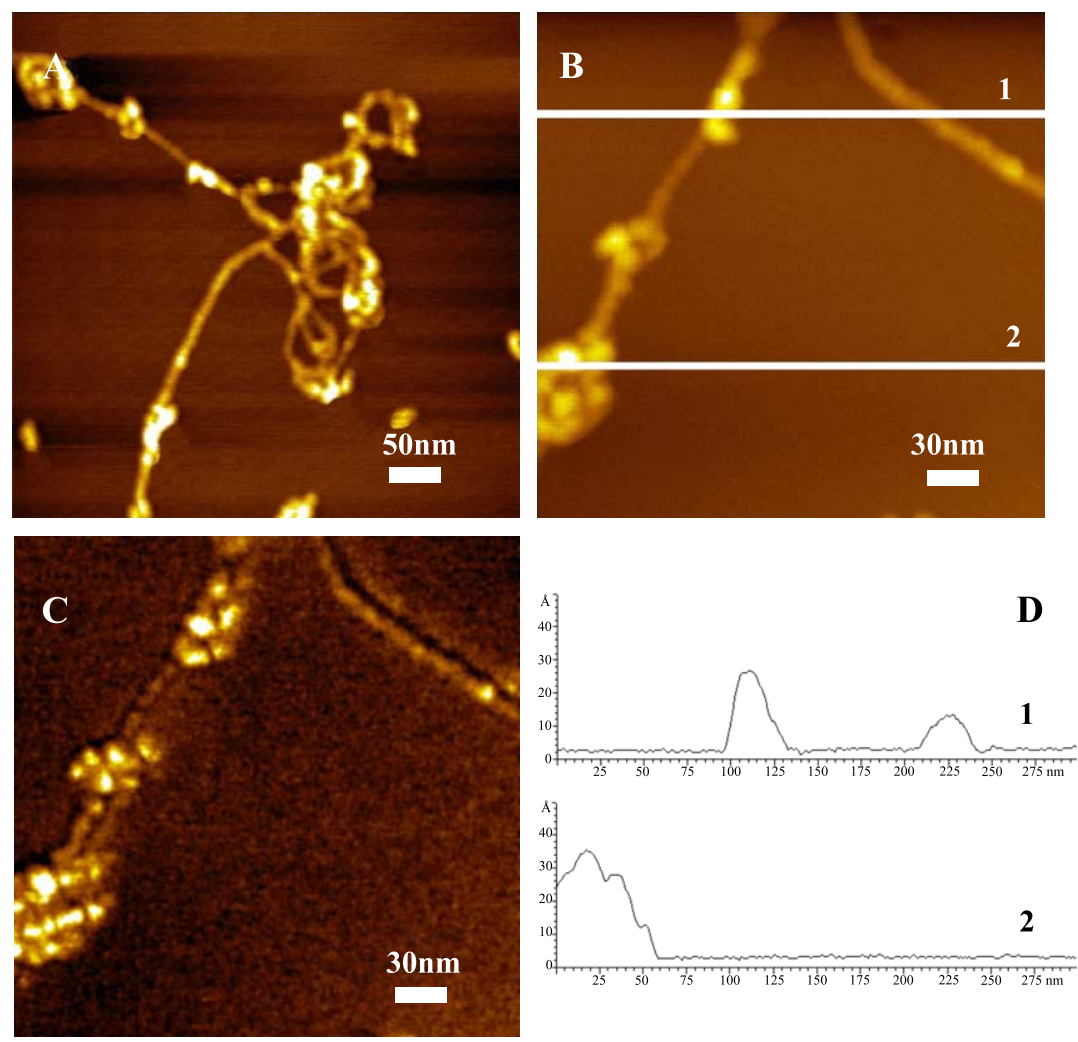

Fig. 5. (A, B) MAC Mode AFM topographical images in air of dsDNA prepared onto HOPG by applying a deposition potential of $+300 \mathrm{mV}$ (vs. AgQRE) during $3 \mathrm{~min}$ to the electrode immersed into $1 \mu \mathrm{g} / \mathrm{ml}$ dsDNA solution $\mathrm{pH}$ 7.0 0.1 M phosphate buffer electrolyte. (C) Phase image recorded simultaneously with the topographic image B. (D) Cross-section profiles through white lines 1 and 2 in the image B.

loops and regions of collapsed and uncollapsed strands coexist together, Fig. 5. Globular aggregates with dimensions between 1.7 and $4 \mathrm{~nm}$ were observed all along the doublestranded chains with a diameter of $1.1 \pm 0.2 \mathrm{~nm}$ Fig. 5D.

\section{Conclusions}

The results suggest that the adsorption of ssDNA and dsDNA at the HOPG surface can be controlled by the applied potential and the electrochemically assisted adsorption provides better attachment of the molecules at the HOPG surface compared with free adsorption. Parts of the molecules interact together by hydrogen bonding during equilibration on the substrate, and hydrophobic interactions and van der Waals forces may also contribute to adsorption on the HOPG electrode. For both ssDNA and dsDNA, the applied potential increased the stability of the adsorbed molecules that bind very tightly, through multiple electrostatic interactions of the phosphate groups, with the positively charged HOPG electrode surface. Due to stronger adsorption of the molecules on the HOPG substrate, the condensed molecules were less compressible by the AFM tip, which explains the higher values of DNA heights compared with free adsorption.

The AFM images MAC Mode AFM offers the possibility of overcoming the difficulties in STM imaging of DNA related to the HOPG grain boundaries and imaging isolated DNA molecules attached to defect-free HOPG surface terraces. The phase images recorded simultaneously with the topographic images enabled the clarification of the morphology of the adsorbed DNA. MAC Mode AFM in an electrochemically controlled environment is capable of resolving the surface morphological structure of nucleic acids adsorbates and contributes to the understanding of the mechanism of adsorption and the nature of DNA-electrode surface interactions.

\section{Acknowledgements}

Financial support from Fundação para a Ciência e Tecnologia (FCT), Post-Doc Grant SFRH /BPD/14425/ 2003 (A.-M. C.P.), POCTI (co-financed by the European Community Fund FEDER), ICEMS (Research Unit 103) and European Projects QLK3-2000-01311 and HPRN-CT2002-00186 are gratefully acknowledged.

\section{References}

[1] W. Saenger, in: Ch.R. Cantor (Ed.), Principles of Nucleic Acid Structure, Springer-Verlag, New York, 1984.

[2] N.C. Seeman, Novel DNA constructions, Annu. Rev. Biophys. Biomol. Struct. 27 (1998) 225-248. 
[3] C. Mao, T.H. LaBean, J.H. Reif, N.C. Seeman, Logical computation using algorithmic self-assembly of DNA triple-crossover molecules, Nature 407 (2002) 493-496.

[4] M.I. Pividori, A. Merkoçi, S. Alegret, Electrochemical genosensor design: immobilization of oligonucleotides onto transducer surfaces and detection methods, Biosens. Bioelectron. 15 (2000) 291-303.

[5] H.G. Hansma, J. Vesenka, C. Siegerist, G. Kelderman, H. Morrett, R.L. Sinsheimer, V. Elings, C. Bustamante, P.K. Hansma, Reproducible imaging and dissection of plasmid DNA under liquid with the atomic force microscope, Science 256 (1992) 1180-1184.

[6] H.G. Hansma, I. Revenko, K. Kim, D.E. Laney, Atomic force microscopy of long and short double-stranded, single-stranded and triple-stranded nucleic acids, Nucleic Acids Res. 24 (1996) $713-720$

[7] A. Sanchez-Sevilla, J. Thimonier, M. Marilley, J. Rocca-Serra, J. Barbet, Accuracy of AFM measurements of the contour length of DNA fragments adsorbed onto mica in air and in aqueous buffer, Ultramicroscopy 92 (2002) 151-158.

[8] R. Schneider, R. Lurz, G. Luder, C. Tolksdorf, A. Travers, G. Muskhelishvili, An architectural role of the Escherichia coli chromatin protein FIS in organising DNA, Nucleic Acids Res. 29 (2001) 5107-5114

[9] O. Medalia, J. Englander, R. Guckenberger, J. Sperling, AFM imaging in solution of protein-DNA complexes formed on DNA anchored to a gold surface, Ultramicroscopy 90 (2001) 103-112.

[10] A. Hamelin, The crystallographic orientation of gold surfaces at the gold aqueous-solution interphases, J. Electroanal. Chem. 142 (1982) 299-316

[11] B.E. Conway, Electrochemical oxide film formation at noble metals as a surface-chemical process, Prog. Surf. Sci. 49 (1995) 351-452.

[12] A.M. Oliveira-Brett, F.-M. Matysik, Voltammetric and sonovoltammetric studies on the oxidation of thymine and cytosine at a glassy carbon electrode, J. Electroanal. Chem. 429 (1997) 95-99.

[13] A.M. Oliveira-Brett, S.H.P. Serrano, J.A.P. Piedade, Electrochemistry of DNA, in: R.G. Compton, G. Hancock (Eds.), Comprehensive
Chemical Kinetics, Applications of Kinetic Modeling, vol. 37, Elsevier, Oxford, UK, 1999, pp. 91-119, Chap. 3.

[14] A.M. Oliveira-Brett, M. Vivan, I.R. Fernandes, J.A.P. Piedade, Electrochemical detection of in situ adriamycin oxidative damage to DNA, Talanta 56 (2002) 959-970.

[15] C.R. Clemmer, Th.P. Beebe Jr., Graphite: a mimic for DNA and other biomolecules in scanning tunneling microscope studies, Science 251 (1991) 640-642.

[16] W.M. Heckl, G. Binning, Domain walls on graphite mimic DNA, Ultramicroscopy 42-44 (1992) 1073-1078.

[17] G.Y. Liu, H. Chang, A.J. Bard, Large scale hexagonal domain structures superimposed on the atomic corrugation of a graphite surface observed by scanning tunneling microscopy, Langmuir 7 (1991) 1139-1142

[18] A.M. Oliveira-Brett, A.-M. Chiorcea, Effect of $\mathrm{pH}$ and applied potential on the adsorption of DNA on highly oriented pyrolytic graphite electrodes. Atomic force microscopy surface characterization, Electrochem. Commun. 5 (2003) 178-183.

[19] A.M. Oliveira Brett, A.-M. Chiorcea, Atomic force microscopy of DNA immobilized onto a highly oriented pyrolytic graphite electrode surface, Langmuir 19 (2003) 3830-3890.

[20] S. Tanaka, Y. Maeda, L.-T. Cai, H. Tabata, T. Kawai, Formation of two-dimensional network structure of DNA molecules on highly oriented pyrolytic graphite surface, Jpn. J. Appl. Phys. 40 (2001) 4217-4220.

[21] M. Argaman, R. Golan, N.H. Thomson, H.G. Hansma, Phase imaging of moving DNA molecules and DNA molecules replicated in the atomic force microscope, Nucleic Acids Res. 25 (1997) 4379-4384.

[22] C. Gaillard, F. Strauss, Eliminating DNA loss and denaturation during storage in plastic microtubes, Am. Clin. Lab. 20 (2001) 52-54.

[23] J.-F. Allemand, D. Bensimon, L. Jullien, A. Bensimon, V. Croquette, pH-dependent specific binding and combing of DNA, Biophys. J. 73 (1997) 2064-2070.

[24] C.M.A. Brett, A.M. Oliveira Brett, Electrochemistry. Principles, Methods, and Applications, Oxford University Press, Oxford, 1993. 